\title{
Come gestire l'ipertensione arteriosa in emodialisi?
}

\author{
Andrea Cavalli, Giuseppe Pontoriero \\ S.C. Nefrologia e Dialisi, Ospedale "Alessandro Manzoni", Lecco
}

\begin{abstract}
Dealing with hypertension in hemodialysis patients
Hypertension is a well-known cardiovascular risk factor also for hemodialysis patients. Despite being common in this patient population, hypertension is often difficult to diagnose and poorly controlled.

Blood pressure variability (BPV) is a new parameter and a predictor of poor outcomes in the general population. A recent observational study in over 11,000 hemodialysis patients showed that high BPV is associated with an increased risk of all-cause mortality, cardiovascular mortality, and first cardiovascular event. High BPV is associated with female gender, black race, obesity, diabetes and/or cardiovascular disease, hemoglobin levels less than $10 \mathrm{~g} / \mathrm{dL}$, high calcium-phosphate product, and use of three or more anti-hypertensive drugs.

Today, the management of hypertension in hemodialysis patients requires more and more care, not only from the diagnostic point of view, but also in prognosis and therapy, especially regarding the non-pharmacological approach.
\end{abstract}

Keywords: Hypertension, Blood pressure variability, Hemodialysis, Mortality, Non-pharmacological treatment

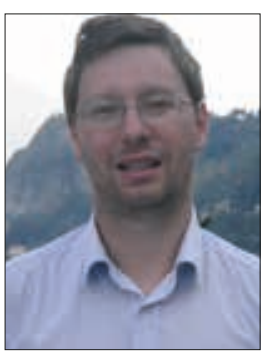

Andrea Cavalli
Dopo aver già trattato nelle scorse rubriche di TN\&D Journal Club $(1,2)$ alcune complicanze e alcuni fattori di rischio cardiovascolare (iperparatiroidismo secondario e anemia) associati all'insufficienza renale cronica (IRC), in questo numero abbiamo deciso di affrontare un altro tipico fattore di rischio cardiovascolare, l'ipertensione arteriosa, vista però dalla prospettiva del paziente emodializzato. In particolare, abbiamo preso spunto dalla recente pubblicazione su un'autorevole rivista di due articoli, comparsi, diciamo così, ad hoc: uno studio osservazionale che introduce nuovi concetti e ipotesi da testare in trial futuri (3) e un'esaustiva review riguardante la gestione dell'ipertensione arteriosa (4).

Accepted: February 25, 2015

Published online: March 5, 2015

Indirizzo per la corrispondenza:

Prof. Giuseppe Pontoriero

S.C. Nefrologia e Dialisi

Ospedale "Alessandro Manzoni"

Via dell'Eremo 9/11

23900 Lecco

g.pontoriero@ospedale.lecco.it

\section{Il significato clinico della variabilità della pressione arteriosa nel paziente emodializzato}

Il lavoro di Shafi et al. (3) parte dalla constatazione di come la maggior parte degli studi abbia analizzato il rapporto tra valori pressori pre-dialitici medi e outcome dei pazienti, anche se è all'ordine del giorno l'evidenza di come i soggetti emodializzati presentino rilevanti variazioni dei valori pressori nel corso della giornata e, ancora più tipicamente, nel periodo intra- e peri-seduta dialitica.

Dati derivanti da precedenti studi hanno evidenziato come la cosiddetta variabilità della pressione arteriosa (BPV) rifletta una rottura dell'omeostasi della pressione arteriosa, derivante da una patologia di tipo micro o macrovascolare in grado di determinare una maggiore suscettibilità alle variazioni di pressione in seguito a stimoli ambientali (5) come, nel caso del soggetto emodializzato, il cambiamento di volume extracellulare, gli inusuali schemi di assunzione della terapia anti-ipertensiva o la possibile dializzabilità dei farmaci (6). In maniera molto pratica, ma facile da comprendere, l'entità di BPV indica quanto ampie siano le variazioni dei valori di pressione (sistolica, rilevata pre-dialisi nel caso in questione) presentate da un soggetto.

I pazienti ipertesi con IRC non in trattamento sostitutivo, rispetto agli ipertesi con normofunzione renale, presentano una maggiore BPV, la quale risulta associata a un più elevato rischio di eventi vascolari come ictus e infarto miocardico (7).

Pertanto, al fine di analizzare i fattori associati alla BPV sistolica pre-dialitica e il suo possibile rapporto con outcome 
clinici nei dializzati, è stato condotto uno studio osservazionale americano comprendente 11.291 pazienti che iniziavano l'emodialisi tra il 2003 e il 2008, con un follow-up mediano di 22 mesi (3). L'età media era di 62 anni, il 55\% dei soggetti era di sesso maschile e il 58\% di razza bianca; una percentuale elevata di soggetti era diabetica (65\%), con malattia cardiovascolare $(56 \%)$ o con scompenso cardiaco (46\%). La pressione sistolica pre-dialitica media era pari a $151 \pm 20 \mathrm{mmHg}$ e coloro che presentavano i più alti valori pressori erano tipicamente più giovani, di sesso femminile, di razza nera e diabetici.

Dopo aver suddiviso i valori di BPV in terzili, emergeva come la più alta BPV fosse associata a sesso femminile, razza nera, presenza di diabete mellito o malattia cardiovascolare (fattori non modificabili), BMI (indice di massa corporea) superiore a 40 , valori di emoglobina inferiori a $10 \mathrm{~g} / \mathrm{dL}$, più elevato prodotto calcio-fosforo e assunzione di almeno tre farmaci anti-ipertensivi (fattori modificabili) (Tab. I). Al contrario, i soggetti che presentavano più bassa BPV avevano un $\mathrm{BMI}$ inferiore a 18.5 , emoglobina superiore a $10 \mathrm{~g} / \mathrm{dL}$, albuminemia più elevata, maggiore deidratazione intra-dialitica e compliance con il peso secco prescritto e una terapia antiipertensiva senza beta-bloccanti o farmaci inibenti il sistema renina-angiotensina (ACE-inibitori e sartani) (Tab. II).

Nel corso di un follow-up mediano di 22 mesi ci sono stati 3.200 decessi (28.3\%): un'elevata BPV era associata a un aumento del rischio di morte, che era pari al $18 \%$ (intervallo di

TABELLA I - Fattori associati ad alta variabilità della pressione arteriosa (BPV) sistolica pre-dialitica

\begin{tabular}{ll}
\hline Non Modificabili & Modificabili \\
\hline Sesso femminile & $\mathrm{BMI}>40$ \\
Razza nera & Emoglobina $<10 \mathrm{~g} / \mathrm{dL}$ \\
$\begin{array}{l}\text { Presenza di diabete } \\
\text { mellito }\end{array}$ & Elevato prodotto Ca x P \\
$\begin{array}{l}\text { Presenza di malattia car- } \\
\text { diovascolare }\end{array}$ & $\begin{array}{l}\text { Assunzione di almeno tre farmaci anti- } \\
\text { ipertensivi }\end{array}$ \\
\hline
\end{tabular}

$\mathrm{BMI}=$ indice di massa corporea $\left(\mathrm{kg} / \mathrm{m}^{2}\right)$.

TABELLA II - Fattori associati a bassa variabilità della pressione arteriosa (BPV) sistolica pre-dialitica

\footnotetext{
- $\mathrm{BMI}<18.5$

- Emoglobina $>10 \mathrm{~g} / \mathrm{dL}$

- Normoalbuminemia

- Maggiore ultrafiltrazione dialitica

- Raggiungimento del peso secco prescritto

- Terapia anti-ipertensiva non comprendente beta-bloccanti o farmaci inibenti il RAS
}

$\mathrm{BMI}=$ indice di massa corporea $\left(\mathrm{kg} / \mathrm{m}^{2}\right) ; \mathrm{RAS}=$ sistema renina-angiotensina . confidenza al 95\%: 13-22\%), per ogni aumento di BPV pari a una deviazione standard (3).

I decessi per causa cardiovascolare sono stati 1.592 (56\% per cardiopatia non ischemica, 25\% per cardiopatia ischemica, $9 \%$ per evento cerebrovascolare e $10 \%$ per altri motivi cardiovascolari). Un'elevata BPV era associata a un maggior rischio di morte anche nel caso di eventi fatali per causa cardiovascolare, con un aumento pari ancora al 18\% (intervallo di confidenza al 95\%: 12-24\%) (3).

Sono stati registrati 4.528 eventi cardiovascolari di cui 3.832 (84.6\%) erano rappresentati da ospedalizzazioni per cardiopatia ischemica (23.5\%), scompenso cardiaco $(25.4 \%)$, eventi cerebrovascolari (14.5\%), aritmie (11.4\%) e arteriopatia periferica (22.5\%). Anche in questo caso, un'elevata BPV era associata a un incremento del rischio di primo evento cardiovascolare, pari al 10\% (3) (Tab. III).

Lo studio di Shafi et al. (3) conferma e amplia i risultati emersi in due precedenti lavori: un'analisi di BPV in oltre 6.000 emodializzati (8) e un'analisi retrospettiva dell'Hemo Study (9).

Pertanto, il messaggio derivante da questo studio osservazionale (e dai precedenti), e quindi da testare con futuri trial randomizzati, è che un'elevata BPV sistolica pre-dialitica va considerata anche negli emodializzati come un fattore di rischio emergente per aumentata mortalità globale ed eventi cardiovascolari fatali e non. Di conseguenza, oltre alla necessità di riconoscerne la presenza nella pratica clinica quotidiana, occorre cercare di ridurne l'entità, così come provare a comprendere le sue correlazioni con le misurazioni pressorie domiciliari, intra-dialitiche o post-dialitiche e i meccanismi che ne stanno alla base (disfunzione endoteliale? Iperattività simpatica?), in quanto possibili target terapeutici (3).

\section{Diagnosi di ipertensione arteriosa nell'emodializzato}

A questo punto è per noi obbligatorio, attraverso la già citata meta-analisi di Agarwal (4), parlare in maniera più ampia della diagnosi e della gestione dell'ipertensione arteriosa nei pazienti emodializzati e trarne alcuni spunti (non scontati) di aggiornamento per la nostra attività dialitica.

Secondo una delle possibili definizioni, la diagnosi di ipertensione arteriosa (10) prevede valori medi di PA pre-dialisi superiori rispettivamente a $150 \mathrm{mmHg}$ (se sistolica) o a $85 \mathrm{mmHg}$ (se diastolica) per almeno una settimana oppure l'utilizzo di una terapia anti-ipertensiva. In accordo con le Linee Guida della National Kidney Foundation Kidney Disease Outcomes Quality Initiative (NKF/KDOQI), si parla di ipertensione in pre-

TABELLA III - Associazioni tra alta variabilità della pressione arteriosa (BPV) sistolica pre-dialitica e outcome

1. Aumentata mortalità $(+18 \%)$

2. Aumentata mortalità cardiovascolare $(+18 \%)$

3. Maggior rischio di primo evento cardiovascolare (+10\%) 
senza di PA superiore a 140/90 $\mathrm{mmHg}$ o a $130 / 80 \mathrm{mmHg}$, se rilevata rispettivamente pre- o post-dialisi (11).

Per quanto riguarda la prevalenza di ipertensione negli emodializzati, vogliamo citare i dati raccolti dal Gruppo di Studio di Ipertensione Arteriosa della Società Italiana di Nefrologia in un recente studio osservazionale che ha coinvolto 4.022 soggetti appartenenti a 77 centri dialisi (12). In accordo con la suddetta definizione della NKF/KDOQI o con l'utilizzo di una terapia mirata, il $70.3 \%$ dei pazienti si poteva definire iperteso e il $57.7 \%$ assumeva farmaci ad hoc, ma solo il $40 \%$ presentava un adeguato controllo pressorio (12).

Tuttavia, la diagnosi di ipertensione nei pazienti emodializzati è spesso difficile, potendo peraltro portare sia a eccessivo sia a insufficiente trattamento, in quanto strettamente legata all'entità dell'incremento ponderale inter-dialitico e all'ultrafiltrazione, che possono rispettivamente rendere conto di elevati (pre-seduta) o bassi (post-seduta) valori pressori anche nello stesso paziente e nel corso dello stesso trattamento emodialitico. Inoltre, occorre considerare come il monitoraggio pressorio inter-dialitico sia scarsamente correlato con i valori rilevati pre- o post-dialisi (13), per cui non è ancora ben chiaro quando sia preferibile rilevare la pressione arteriosa (PA) nel soggetto emodializzato.

La PA media (o mediana, ancora più facilmente determinabile) durante la seduta emodialitica di metà settimana rappresenta un dato facilmente raccoglibile al letto del paziente e migliore, rispetto ai soli parametri di attacco e stacco, nello stimare la "reale" PA domiciliare. Infatti, valori superiori a $140 / 90 \mathrm{mmHg}$ indicano un quadro di ipertensione arteriosa al domicilio (14).

Tuttavia, la metodica più pratica, semplice ed economica per porre diagnosi e gestire la PA nei pazienti in emodialisi è rappresentata dalla "banale" misurazione periodica domiciliare della $\mathrm{PA}$, in quanto più strettamente correlata con quella rilevata attraverso un monitoraggio delle 24 ore (rispetto alle rilevazioni pre- e post-dialitiche), oltre a essere in grado di evidenziare le modifiche pressorie secondarie all'ultrafiltrazione intra-seduta e a essere riproducibile nel corso del tempo $(15,16)$.

Inoltre, la PA domiciliare si è dimostrata superiore a quella rilevata in dialisi nel predire la presenza di ipertrofia ventricolare sinistra, eventi cardiovascolari e mortalità $(17,18)$.

Considerando che la PA presenta un aumento medio di $4 \mathrm{mmHg}$ ogni 10 ore dalla fine del trattamento emodialitico, non sono consigliabili rilevazioni appena prima della seduta o subito dopo. Al contrario, si può consigliare di misurare la PA due volte al giorno (la mattina e prima di coricarsi), a partire dalla seduta di metà settimana e per 4 giorni. In questo modo si può diagnosticare e gestire adeguatamente l'ipertensione arteriosa nell'emodializzato, che, nel lungo termine, potrà proseguire con tali modalità di monitoraggio, eseguendole una volta al mese (19).

Basandosi sulle misurazioni pressorie domiciliari, il target pressorio a cui ambire può essere desunto da quello definito dalle Linee Guida dell'American Heart Association (cioè una
PA inferiore a $135 / 85 \mathrm{mmHg}$ (20), per cui valori pressori al di sotto di $140 / 90 \mathrm{mmHg}$ nel periodo inter-dialitico possono sembrare ragionevoli negli emodializzati, anche se non esistono a oggi valori di riferimento specifici derivati da studi mirati.

Anche nell'emodializzato, la metodica migliore per la diagnosi di ipertensione arteriosa, oltre che per la sua elevata correlazione con la prognosi del paziente, è però rappresentata dal monitoraggio pressorio ambulatoriale, specialmente se eseguito nell'arco dell'intero intervallo inter-dialitico di 44 ore. Occorre ricordare che solitamente rileva una riduzione di $2.5 \mathrm{mmHg}$ ogni 10 ore trascorse dal termine della seduta (21, 22) (Tab. IV).

Diversa rispetto all'ipertensione tout court, ma assolutamente da menzionare, è l'ipertensione intra-dialitica, definita come un aumento paradosso della PA durante il trattamento emodialitico, caratterizzante circa il $10-15 \%$ dei soggetti e associata ad aumentate ospedalizzazione e mortalità $(23,24)$.

\section{Terapia non farmacologica dell'ipertensione arteriosa nell'emodializzato}

Per quanto riguarda il trattamento dell'ipertensione arteriosa, vogliamo brevemente riportare in questa sede soltanto i possibili approcci non farmacologici (Tab. V), lasciando eventualmente spazio per i farmaci anti-ipertensivi a una futura rubrica di TN\&D.

TABELLA IV - Possibili metodi per rilevare la pressione arteriosa nel soggetto emodializzato, in ordine decrescente di qualità, per permettere una corretta stima della "reale" pressione arteriosa

- Monitoraggio pressorio ambulatoriale (meglio se per 44 ore)

Pressione arteriosa domiciliare

Pressione arteriosa media (o mediana) durante la seduta emodialitica di metà settimana

Pressione arteriosa pre- e post-dialitica

TABELLA V-Approccio terapeutico non farmacologico all'ipertensione arteriosa nell'emodializzato

\footnotetext{
- Corretta diagnosi di ipertensione

- Dieta iposodica (1.5-2 g/die)

- Aumentata attività fisica e/o calo ponderale se obesità

- Abolizione del fumo

- Valutazione del bilancio del sodio

Riduzione del peso secco

Aumento della durata della seduta emodialitica

Valutazione di eventuali terapie concomitanti (ad es., eritropoietina) e comorbidità (ad es., apnee notturne)
} 
Gli approcci non farmacologici al trattamento dell'ipertensione arteriosa sono in parte sovrapponibili a quelli attuabili nella popolazione generale. Infatti, il calo ponderale in caso di obesità, l'abolizione del fumo e l'esercizio fisico sono sempre da raccomandare anche nei nostri pazienti. Lo stesso si può dire per la dieta iposodica, in quanto permette di contenere gli incrementi inter-dialitici e di raggiungere il peso secco: attualmente le Linee Guida raccomandano un apporto di sodio non superiore a 1.5-2 g/die (25).

L'utilizzo di concentrazioni di sodio nel dialisato più alte rispetto alla natremia pre-dialitica può determinare un bilancio sodico positivo e un aumento del senso di sete e degli incrementi ponderali inter-dialitici (se il paziente non riesce a frenare lo stimolo a bere), con una progressiva espansione dei compartimenti liquidi dell'organismo e un aumento della pressione arteriosa (oltre al rischio di edema polmonare). Pertanto la personalizzazione della concentrazione di sodio nel bagno di dialisi rappresenta un elemento cardine nella gestione dell'ipertensione arteriosa nell'emodializzato (25).

L'altro cardine del trattamento dell'ipertensione arteriosa nell'emodializzato è rappresentato dalla riduzione del peso secco, che si può ritenere utile anche in quei soggetti che non presentano evidenti segni di ritenzione idrosodica (26). In caso di insorgenza di una sintomatologia intra-dialitica (ipotensione, crampi e vomito, per esempio), oltre alla programmazione solo di una "gentile" (ma progressiva) riduzione del peso corporeo (2-3 hg per seduta), sarà ovviamente da considerare l'opportunità di aumentare la durata del trattamento emodialitico o la sua frequenza (4). D'altro canto, occorre ricordare come la riduzione del peso secco possa contribuire a determinare possibili gravi complicanze: trombosi dell'accesso vascolare, scomparsa della funzione renale residua ed episodi di ipotensione intra-dialitica, i quali possono a loro volta essere responsabili di ipoperfusione cerebrale, cardiaca o mesenterica (4).

Anche nel caso dell'ipertensione intra-dialitica, la personalizzazione della concentrazione di sodio nel dialisato e la riduzione del peso secco si sono dimostrati validi provvedimenti (24).

In alcuni casi occorre invece prendere in esame altre possibili cause alla base di un aumento dei valori pressori, come la concomitante terapia con eritropoietina o la presenza della sindrome delle apnee notturne (4).

Speriamo che questa breve discussione abbia permesso di fare chiarezza su alcuni nuovi parametri, di fatto non solo sperimentali, ma anche squisitamente clinici come la BPV, importanti per i pazienti emodializzati, oltre che aver richiamato la nostra attenzione sulle migliori modalità per la diagnosi e la gestione di un così frequente, ma spesso misconosciuto e mal-trattato, fattore di rischio cardiovascolare.

\section{Disclosures}

Financial support: No financial support was received for this submission.

Conflict of interest: The authors have no conflict of interest.

\section{Bibliografia}

1. Cavalli A, Pontoriero G. Studio EVOLVE: un'altra delusione per i Nefrologi. Giornale di Tecniche Nefrologiche e Dialitiche 2013;25(3):261-5.

2. Cavalli A, Pontoriero G. Quali sono le ultime novità in tema di terapia marziale. Giornale di Tecniche Nefrologiche e Dialitiche 2014;26(3):296-300

3. Shafi T, Sozio SM, Bandeen-Roche KJ, et al. Predialysis systolic BP variability and outcomes in hemodialysis patients. J Am Soc Nephro 2014;25:799-809.

4. Agarwal R, Flynn J, Pogue V, et al. Assessment and management of hypertension in patients on dialysis. J Am Soc Nephrol 2014;25:1630-46.

5. Mancia G. Prognostic value of long-term blood pressure variability: the evidence is growing. Hypertension 2011;57:141-3.

6. Flythe JE, Kunaparaju S, Dinesh K, et al. Factors associated with intradialytic systolic blood pressure variability. Am J Kidney Dis 2012;59:409-18.

7. Rothwell PM, Howard SC, Dolan E, et al. Prognostic significance of visit-to-visit variability, maximum systolic blood pressure, and episodic hypertension. Lancet 2010;375:895-905.

8. Flythe JE, Inrig JK, Shafi T, et al. Association of intradialytic blood pressure variability with increased all-cause and cardiovascula mortality in patients treated with long-term hemodialysis. Am J Kidney Dis 2013;61(6):966-74.

9. Chang TI, Flythe JE, Brunelli SM, et al. Visit-to-visit systolic blood pressure variability and outcomes in hemodialysis. J Hum Hypertens 2014;28(1):18-24

10. Agarwal R, Nissenson AR, Batlle D, Coyne DW, Trout JR, Warnock DG. Prevalence, treatment, and control of hypertension in chronic hemodialysis patients in the United States. Am J Med 2003;115:291-7.

11. K/DOQI Workgroup. K/DOQI clinical practice guidelines for cardiovascular disease in dialysis patients. Am J Kidney Dis 2005;45 (Suppl. 3): S1-153.

12. Del Vecchio L, Lusenti T, Del Rosso G, et al. Prevalence of hypertension in a large cohort of Italian hemodialysis patients: results of a crosssectional study. J Nephrol 2013;26(4):745-54.

13. Agarwal R, Peixoto AJ, Santos SF, Zoccali C. Pre- and postdialysis blood pressures are imprecise estimates of interdialytic ambulatory blood pressure. Clin J Am Soc Nephrol 2006;1:389-98.

14. Agarwal R, Metiku T, Tegegne GG, et al. Diagnosing hypertension by intradialytic blood pressure recordings. Clin J Am Soc Nephrol 2008;3:1364-72

15. Agarwal R, Andersen MJ, Bishu K, Saha C. Home blood pressure monitoring improves the diagnosis of hypertension in hemodialysis patients. Kidney Int 2006;69:900-6.

16. Agarwal R, Satyan S, Alborzi $P$, et al. Home blood pressure measurements for managing hypertension in hemodialysis patients. Am J Nephrol 2009;30:126-34.

17. Agarwal R, Brim NJ, Mahenthiran J, AndersenMJ, SahaC. Out-ofhemodialysis unit blood pressure is a superior determinant of left ventricular hypertrophy. Hypertension 2006;47:62-8.

18. Moriya $\mathrm{H}$, Oka $\mathrm{M}$, Maesato $\mathrm{K}$, et al. Weekly averaged blood pressure is more important than a single-point blood pressure measurement in the risk stratification of dialysis patients. Clin J Am Soc Nephro 2008;3:416-22.

19. Agarwal R, AndersenMJ, Light RP. Location not quantity of blood pressure measurements predicts mortality in hemodialysis patients. Am J Nephrol 2008;28:210-7.

20. Pickering TG, Miller NH, Ogedegbe G, Krakoff LR, Artinian NT, Goff D. Call to action on use and reimbursement for home blood pressure monitoring: a joint scientific statement from the American Heart Association, American Society Of Hypertension, and Preventive Cardiovascular Nurses Association. Hypertension 2008;52:10-29.

21. Thompson AM, Pickering TG. The role of ambulatory blood pressure monitoring in chronic and end-stage renal disease. Kidney Int 2006;70:1000-7.

22. Agarwal R. Blood pressure and mortality among hemodialysis patients Hypertension 2010;55:762-8.

23. Inrig JK. Intradialytic hypertension: A less recognized cardiovascular complication of hemodialysis. Am J Kidney Dis 2010;55:580-9.

24. Locatelli F, Cavalli A, Tucci B. The growing problem of intradialytic hypertension. Nat Rev Nephrol 2010;6(1):41-8.

25. Kooman JP, van der Sande F, Leunissen K, Locatelli F. Sodium balance in hemodialysis therapy. Semin Dial 2003;16:351-5.

26. Agarwal R, Alborzi P, Satyan S, Light RP. Dry-weight reduction in hypertensive hemodialysis patients (DRIP): a randomized, controlled trial. Hypertension 2009;53:500-7. 